\title{
Usefulness of measuring radiographic density of axis vertebra in patients at risk of osteoporosis: $A$ cone-beam computed tomography study
}

\author{
Mustafa Alkhader ${ }^{1}$, Mohammad S. Alrashdan ${ }^{1}$, Yousef Khader ${ }^{2}$
}

Correspondence: Dr. Mustafa Alkhader

Email: mmalkhader@just.edu.jo

\begin{abstract}
'Department of Oral Medicine and Oral Surgery, Faculty of Dentistry, Jordan University of Science and Technology, Irbid, Jordan,

${ }^{2}$ Department of Community Medicine, Public Health and Family Medicine, Faculty of Medicine, Jordan University of Science and Technology, Irbid, Jordan
\end{abstract}

\section{ABSTRACT}

Objective: Using cone-beam computed tomography (CBCT) images, the aim of the study was to evaluate the usefulness of measuring radiographic density of the axis vertebra (RDAV) in patients at risk of osteoporosis. Materials and Methods: Two hundred and forty-seven old patients (109 males and 138 females) aged between 50 and 80 years (mean age: $59.68 \pm 7.27$ ) were examined by CBCT. Using InVivoDental, v. 5.0 (Anatomage Inc., San Jose, CA), RDAV and mental index (MI) were measured twice and correlated using Pearson's correlation coefficients. Patients were divided into two categories: high risk and low risk of osteoporosis using $3.1 \mathrm{~mm}$ of MI as a cutoff value, and the mean value of RDAV was compared and correlated using independent samples' $t$-test and regression analysis. Receiver-operating characteristic (ROC) curve analysis was also used to examine the predictive power of RDAV. Results: The mean value of RDAV was moderately correlated with MI $(r=0.32)$, and in patients at low risk of osteoporosis, the mean value of RDAV was significantly higher than in patients at high risk of osteoporosis. In multivariate binary logistic regression, the odds of being at risk of osteoporosis decreased by $1 \%$ with one unit increase in RDAV (odds ratio $=0.988,95 \%$ confidence interval: $0.983-0.993 ; P<0.005$ ). ROC analysis showed that the mean value of RDAV had a high predictive power for predicting patients at risk of osteoporosis (area under the curve $=0.761$ for females and 0.649 for males). Conclusions: Measuring RDAV is considered useful in predicting patients at risk of osteoporosis.

Key words: Axis vertebra, cone beam computed tomography, osteoporosis, radiographic density

\section{INTRODUCTION}

Osteoporosis is a systemic disorder characterized by the depletion of bone mass with microarchitectural deterioration of bone tissue, resulting in a decrease in bone mineral density (BMD) and a predisposition to fragility fractures. ${ }^{[1,2]}$ The resultant mortality, morbidity, and financial implications are becoming a major health-care burden worldwide. It is estimated

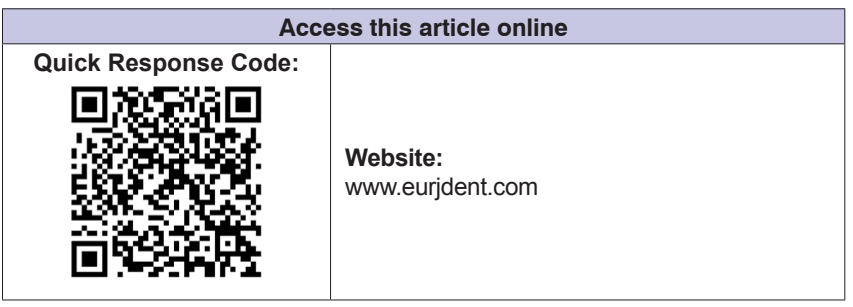

that one in three women and one in six men will suffer at least one osteoporotic fracture during their lifetime. ${ }^{[3]}$ Cross-sectional studies have consistently found that osteoporosis is underdiagnosed both in the general population and in high-risk groups. ${ }^{[2]}$

This is an open access journal, and articles are distributed under the terms of the Creative Commons Attribution-NonCommercial-ShareAlike 4.0 License, which allows others to remix, tweak, and build upon the work non-commercially, as long as appropriate credit is given and the new creations are licensed under the identical terms.

For reprints contact: reprints@medknow.com

How to cite this article: Alkhader M, Alrashdan MS, Khader $\mathrm{Y}$ Usefulness of measuring radiographic density of axis vertebra in patients at risk of osteoporosis: A cone-beam computed tomography study. Eur J Dent 2018;12:496-501.

DOI: 10.4103/ejd.ejd_375_17 
Dual-energy X-ray absorptiometry (DXA) is currently considered the gold standard for the evaluation of BMD ${ }^{[4]}$ DXA is used to measure BMD at the hip, neck of femur, vertebrae, and wrist. DXA provides the patient's T-score, which is the BMD value compared with that of control subjects who are young, healthy adults at the peak of their BMD. ${ }^{[5]}$ Osteoporosis is defined as BMD $>2.5$ standard deviations below the mean of young reference populations. ${ }^{[6]}$

Despite being widely used, DXA test is not without limitations. The inability to assess altered bone quality or to predict the risk of fracture in the osteopenic group, which is rather more common than the nonosteoporotic group ${ }^{[2]}$ and the inability to account for individual risk of falling, which has a substantial impact on the risk of fractures, ${ }^{[7]}$ collectively reduces the predictive value of such a test. Moreover, DXA is not universally accessible in all health-care systems. Therefore, the search for other tools capable of replacing DXA in differentiating high-risk populations by employing simple and more accessible methods is underway and could help bridge the current diagnostic gap in osteoporosis in the near future.

Several studies have shown the feasibility of dental panoramic radiography, and more recently, cone-beam computed tomography (CBCT) in the prediction of osteoporosis in high-risk populations..$^{[8-14]}$

Qualitative and quantitative indices have been developed to assess radiographic density based on dental panoramic radiographs and the validity of these indices has been well established in literature. ${ }^{[15-19]}$ Such indices include the mandibular cortical index, mental index (MI), panoramic mandibular index, gonial index, and antigonial index among others. These indices have shown significant age and gender differences as well as differences between high-risk populations and controls. ${ }^{[17,20-24]}$ Some reports have found certain indices to be superior to others in their predictive values. ${ }^{[25]}$

The association between mandibular indices and radiographic density measured in vertebrae was investigated in several studies. ${ }^{[8-10,13-16,18,24-26]}$ In a recent report, ${ }^{[26]} \mathrm{CBCT}$-derived radiographic density of cervical vertebrae, as measured by CBCT viewer program, was shown to significantly correlate with osteoporosis prediction assessed through DXA. Accordingly, it appears plausible that the value of CBCT-derived radiographic density of the cervical vertebrae in the diagnosis of osteoporosis could also be tested through its correlation with the aforementioned mandibular indices. Therefore, this study was conducted to evaluate the usefulness of measuring radiographic density of the axis vertebra (RDAV) in patients at risk of osteoporosis and to test the RDAV correlation with the MI.

\section{MATERIALS AND METHODS}

\section{Patients}

In our retrospective study, CBCT images for all patients aged 50 years and more were retrieved and evaluated from our CBCT database. All patients underwent CBCT examination for dental implant treatment and other dental purposes at our dental radiology clinic between January 2011 and January 2017. After excluding cases with artifacts or pathology affecting measurements of RDAV or MI, the number of cases included were 247 (109 males and 138 females) aged between 50 and 80 years (mean age $59.68 \pm 7.27$ ). The study protocol was approved by our Institutional Ethical Review Board (no. 447/2016).

\section{Cone-beam computed tomography examination}

As a CBCT apparatus, KODAK 9500 Cone Beam 3D System (Carestream, Rochester, NY, USA) with flat panel detector was used. The imaging area of CBCT is a cylinder with a height of $15-20.6 \mathrm{~cm}$ and a diameter of 9-18 cm providing isotropic cubic voxels with sides approximating $0.2-0.3 \mathrm{~mm}$. Only cases examined with $0.2 \mathrm{~mm}$ of voxel size were included in the study. The exposure parameters were: $90 \mathrm{kV}$ as a tube voltage, $10 \mathrm{~mA}$ as a tube current, and $10.8 \mathrm{~s}$ as an exposure time.

Examinations were performed by $360^{\circ}$ rotation in the occlusal position with the patient standing and closing their teeth.

\section{Images}

One calibrated oral radiologist (MA) with 10 years of experience with CBCT, using InVivoDental, v. 5.0 (Anatomage Inc., San Jose, CA) for measuring RDAV and MI twice within 2 months interval.

For measuring RDAV, a coronal section in the middle of axis vertebra was used. To obtain the coronal section in the middle of axis vertebra, the middle of axis vertebra was localized on both of axial and sagittal images using associated colored bars, then using the distance measurement tool, $8 \mathrm{~mm} \times 16 \mathrm{~mm}$ rectangular area of interest was drawn on the axis vertebra starting from the base of the dens toward the body of the axis, then 
Hounsfield Units (HU) measurement tool was used to generate density value for the corresponding vertebra. HU measurement tool showed three density values at each vertebra (minimum, mean, and maximum). However, we only considered the mean density value for analysis [Figures 1a, 2a,3a].

Before deciding the dimensions of area of interest $(8 \mathrm{~mm} \times 16 \mathrm{~mm})$, a pilot study was conducted, and 20 coronal images in the middle of 20 different axis vertebrae were used to draw the largest possible area of interest without including the outer cortex. $8 \mathrm{~mm} \times 16 \mathrm{~mm}$ rectangular area of interest was the best according to previous criteria and consequently used for all patients.

For measuring MI (inferior cortical width of the mandible at mental foramen), arch section module was utilized and a cross-sectional image in the middle of each mental foramen was used. To obtain a cross-sectional image at the middle of mental foramen, the middle of mental foramen was localized on the corresponding panoramic image using associated longitudinal bar, then using the distance measurement tool, the inferior cortical width of the mandible was measured at each mental foramen following the example of Koh and Kim. ${ }^{[27]}$ However, we only considered the mean MI value for analysis [Figures 1b, c-3b, c]. According to a multi-center study with large sample size, ${ }^{[28]}$ patients with the thinnest $\mathrm{MI}(\leq 3 \mathrm{~mm})$ had the highest likelihood of osteoporosis. Therefore, $3.1 \mathrm{~mm}$ of MI was set as a cutoff value.

All images were evaluated on a high definition LCD display, and window settings were fixed for all cases.

\section{Statistical analysis}

Data were analyzed using SPSS version 20 (SPSS Inc., Chicago Illinois, USA). Data were described using means and percentages. Pearson's correlation coefficient was used to determine intrarater correlation between first and second-time measurements of RDAV and MI and the correlation between RDAV and MI. Using $3.1 \mathrm{~mm}$ of MI as a cutoff value, patients were divided into low and high risk of osteoporosis. Independent $t$-test was used to compare means of continuous variables between those with low and high risk of osteoporosis. The association between RDAV and MI after adjusting for age and gender was tested in linear regression analysis when MI is continuous and binary logistic regression when MI is dichotomous. Receiver operating characteristics (ROCs) analysis was used to test the ability of RDAV to predict the risk

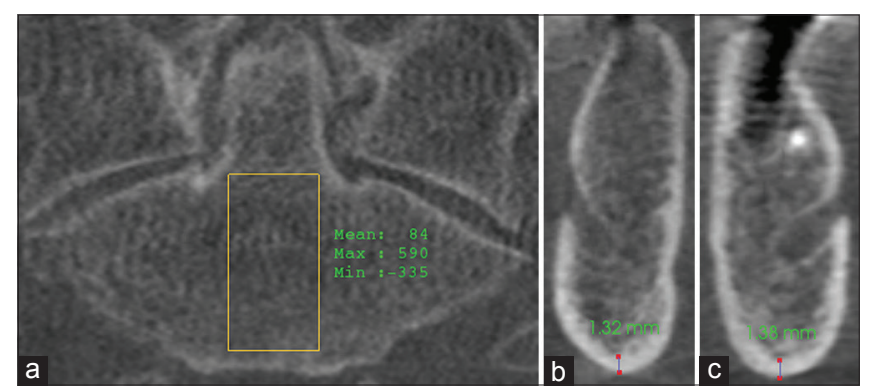

Figure 1: Images of a patient at high risk of osteoporosis showing; radiographic density of axis vertebra (a), mental index at right side of the mandible (b), and mental index at left side of the mandible (c)
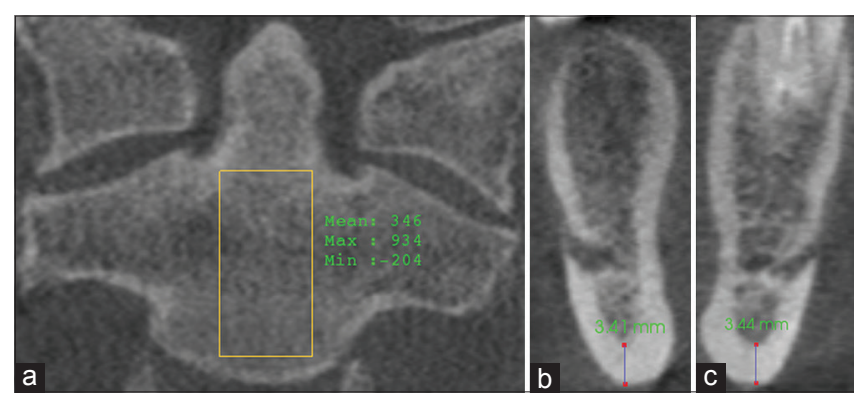

Figure 2: Images of a patient at low risk of osteoporosis showing; radiographic density of axis vertebra (a), mental index at right side of the mandible (b), and mental index at left side of the mandible (c)

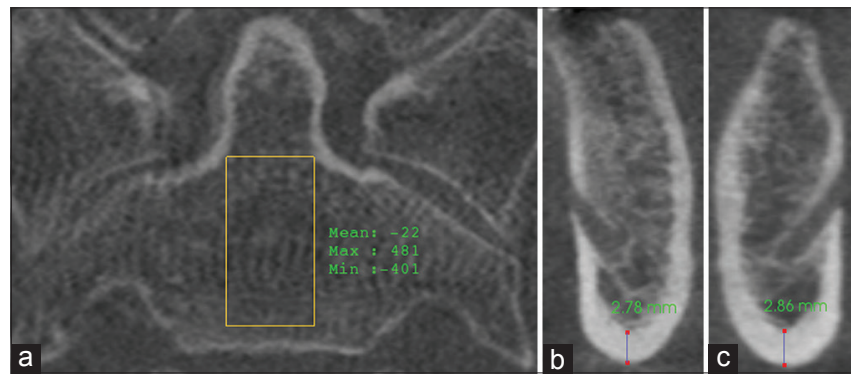

Figure 3: Images of a patient at high risk of osteoporosis showing radiographic density of axis vertebra (a), mental index at right side of the mandible (b), and mental index at left side of the mandible (c)

of osteoporosis. $P<0.05$ was considered statistically significant.

\section{RESULTS}

This study included a total of 247 persons. Theintrarater correlations for RDAV $(r=0.99)$ and $\mathrm{MI}(r=0.98)$ were very strong. Therefore, the two readings for each measurement were averaged to be used for further analysis. The correlation between average RDAV and average MI was moderate $(r=0.32)$.

Patients were divided into high risk and low risk of osteoporosis (34 females and 18 males versus 104 females and 91 males respectively) using $3.1 \mathrm{~mm}$ of MI as a cutoff value. For females only, the mean 
age was significantly higher for females at high risk of osteoporosis compared to females at low risk of osteoporosis (64.0 [7.9] year vs. 58.4 [6.4] year; $P<0.005)$. For males and females, the mean value of RDAV and MI was significantly higher in patients at low risk of osteoporosis compared to those at high risk of osteoporosis [Table 1]. After adjusting for the effects of age and gender, the mean value of $\operatorname{RDAV}(F=23.8$, $P<0.005)$ remained significantly higher in patients at low risk of osteoporosis. In multivariate binary logistic regression, the odds of being at risk of osteoporosis decreased by $1 \%$ with one unit increase in RDAV (odds ratio $=0.988,95 \%$ confidence interval: $0.983-0.993 ; P<0.005)$. ROC analysis showed that the mean value of RDAV (area under the curve $=0.761$ for females and 0.649 for males) had a high predictive power to predict osteoporosis.

\section{DISCUSSION}

In this study, we evaluated the usefulness of RDAV in predicting osteoporosis in elderly patients. According to Jonasson et al., ${ }^{[29]}$ mandibular cortical thickness decreased significantly after age of 50, and more fractures were shown. Therefore, patients above 50 years old are at risk of osteoporosis and possibility to have low $\mathrm{MI}$ is high as shown in similar studies. ${ }^{[24,30]}$

DXA is considered the modality of choice for predicting patients at risk of osteoporosis. However, DXA has several limitations, especially in obese patients and/or patients with degenerative changes in the spine. ${ }^{[4]}$ Furthermore, DXA is not available in routine dental practice; therefore, other imaging modalities with several advantages over DXA are being used in medicine, like the single slice CT for example. ${ }^{[4]}$

\begin{tabular}{|c|c|c|c|c|c|}
\hline & \multicolumn{4}{|c|}{ Mean $\pm S D$} & \multirow[t]{2}{*}{$P$} \\
\hline & \multicolumn{2}{|c|}{$\begin{array}{l}\text { High risk of } \\
\text { osteoporosis }\end{array}$} & \multicolumn{2}{|c|}{$\begin{array}{l}\text { Low risk of } \\
\text { osteoporosis }\end{array}$} & \\
\hline \multicolumn{6}{|l|}{ Females } \\
\hline Age & 64.0 & 7.9 & 58.4 & 6.4 & $<0.005$ \\
\hline RDAV & 95.8 & 70.8 & 176.2 & 87.0 & $<0.005$ \\
\hline $\mathrm{Ml}$ & 2.5 & 0.4 & 3.9 & 0.5 & $<0.005$ \\
\hline \multicolumn{6}{|l|}{ Males } \\
\hline Age & 60.9 & 7.5 & 59.3 & 7.3 & 0.390 \\
\hline RDAV & 115.5 & 71.8 & 164.0 & 74.1 & 0.015 \\
\hline MI & 2.7 & 0.3 & 4.0 & 0.6 & $<0.005$ \\
\hline
\end{tabular}

Searching the literature revealed a single study which investigated the usefulness of radiographic density of cervical vertebrae in predicting patients at risk of osteoporosis. ${ }^{[26]}$ The researchers in this particular report focused on the correlation of radiographic density of first and second cervical vertebrae with DXA results of lumbar vertebrae and femoral neck, and MI was not included in the analysis. The results confirmed the usefulness of measuring radiographic density of cervical vertebrae. However, the researchers used one CBCT device with its associated software, and in all cases, the software measured radiographic density of cervical vertebrae by including the outer cortex in measurements. From our experience, the outer cortex is commonly associated with bone changes and/or degenerative changes and including this part in analysis will affect the final radiographic density measurements. To overcome this shortcoming, we have selected an area in the middle of axis vertebra and we excluded the cortex from analysis. Moreover, utilizing large cervical vertebrae like axis vertebra, in the current study, has allowed a large area of interest $(8 \mathrm{~mm} \times 16 \mathrm{~mm})$ to be drawn without including the outer cortex in analysis. From a feasibility point of view, it is important to note that axis vertebra is present in all CBCT images taken with medium field of view.

Despite the differences in methodology and analysis, our results were in line with those of Barngkgei et al., [26] confirming that RDAV is useful in predicting patients at risk of osteoporosis and it is significantly lower in patients with osteoporosis.

To confirm the usefulness of RDAV in predicting patients at high risk of osteoporosis, we have also included the area under the ROC curve in our analysis and found that it was 0.649 and 0.761 for males and females, respectively. This indicates high predictive power of RDAV. Similar results were obtained using MI measurements for predicting osteoporosis. ${ }^{[24,25]}$ Therefore, RDAV might be recommended to be used instead of MI when mandibular bone is not shown in patient images for any reason.

Among radiographic indices used to predict patients at risk of osteoporosis is MI. MI is one of the most accurate indices to be studied. ${ }^{[16,19,25,28]}$ Even in studies which measured MI on cross-sectional CBCT images, ${ }^{[13,14]}$ MI was significantly higher in patients at low risk of osteoporosis. Data reported in the current study did not deviate from these findings. It is noteworthy, however, that we did not 
consider mandibular cortical index or panoramic mandibular index in the analysis, since the first is subjectively analyzed depending on the experience of the observer and the latter is affected by the location of mental foramen; normal variations in the location of mental foramen are expected and might affect the results.

Considering the effect of gender and age on MI, we have demonstrated that MI values were higher in males and inversely related to age. This appears consistent with relevant literature. ${ }^{[15,17,23,24]}$ Interestingly, Dutra et al. ${ }^{[31]}$ demonstrated greater MI measurements for older males in their report, which could be the result of specifications unique to the ethnicity studied. Moreover, only through a longitudinal study design would it be possible to determine how age would affect the MI within the same cohort. The finding that the mean age was significantly higher for females at high risk of osteoporosis compared to females at low risk of osteoporosis in our study could be attributed to the fact that more females in menopause or postmenopause are present in the older age group.

Dental status or the number of remaining teeth in older patients might be associated with osteoporosis. ${ }^{[21,22]}$ All patients included in the current study had multiple missing teeth except five patients who had single missing tooth; therefore, the relationship between RDAV, MI, and dental status could not be established.

In this study, all patients with $\mathrm{MI} \leq 3 \mathrm{~mm}$ were considered at high risk of osteoporosis as validated in previous studies; ${ }^{[21,28,30]}$ however, this cutoff value of MI might be race or ethnic group dependent. ${ }^{[2]} \mathrm{A}$ limitation of the current study is that the accuracy of the applied cutoff value was not tested due to lack of other tests such as DXA or quantitative CT. Finally, the dimensions of area of interest used in this study might not be precisely applicable to images of patients from different ethnic groups.

\section{CONCLUSIONS}

The findings of this study indicate that RDAV could represent a useful tool in predicting patients at risk of osteoporosis.

\section{Acknowledgment}

We are thankful for the Deanship of Scientific Research in Jordan University of Science and Technology for their approval and support in conducting the research.
Financial support and sponsorship Nil.

\section{Conflicts of interest}

There are no conflicts of interest.

\section{REFERENCES}

1. Friedman AW. Important determinants of bone strength: Beyond bone mineral density. J Clin Rheumatol 2006;12:70-7.

2. Høiberg MP, Rubin KH, Hermann AP, Brixen K, Abrahamsen B. Diagnostic devices for osteoporosis in the general population: A systematic review. Bone 2016;92:58-69.

3. Kanis JA, Borgström F, Compston J, Dreinhöfer K, Nolte E, Jonsson L, et al. Scope: A scorecard for osteoporosis in Europe. Arch Osteoporos 2013;8:144.

4. Link TM, Lang TF. Axial QCT: Clinical applications and new developments. J Clin Densitom 2014;17:438-48.

5. Chan CK, Mason A, Cooper C, Dennison E. Novel advances in the treatment of osteoporosis. Br Med Bull 2016;119:129-42.

6. Schousboe JT, Shepherd JA, Bilezikian JP, Baim S. Executive summary of the 2013 International Society for Clinical Densitometry Position Development Conference on bone densitometry. J Clin Densitom 2013;16:455-66.

7. Seeman E, Delmas PD. Bone quality - The material and structural basis of bone strength and fragility. N Engl J Med 2006;354:2250-61.

8. Taguchi A, Suei Y, Ohtsuka M, Otani K, Tanimoto K, Ohtaki M, et al. Usefulness of panoramic radiography in the diagnosis of postmenopausal osteoporosis in women. Width and morphology of inferior cortex of the mandible. Dentomaxillofac Radiol 1996;25:263-7.

9. Taguchi A, Ohtsuka M, Nakamoto T, Naito K, Tsuda M, Kudo Y, et al. Identification of post-menopausal women at risk of osteoporosis by trained general dental practitioners using panoramic radiographs. Dentomaxillofac Radiol 2007;36:149-54.

10. Gaur B, Chaudhary A, Wanjari PV, Sunil M, Basavaraj P. Evaluation of panoramic radiographs as a screening tool of osteoporosis in post menopausal women: A cross sectional study. J Clin Diagn Res 2013;7:2051-5.

11. Barngkgei I, Al Haffar I, Khattab R. Osteoporosis prediction from the mandible using cone-beam computed tomography. Imaging Sci Dent 2014;44:263-71.

12. Gomes CC, de Rezende Barbosa GL, Bello RP, Bóscolo FN, de Almeida SM. A comparison of the mandibular index on panoramic and cross-sectional images from CBCT exams from osteoporosis risk group. Osteoporos Int 2014;25:1885-90.

13. Güngör E, Yildirim D, Çevik R. Evaluation of osteoporosis in jaw bones using cone beam CT and dual-energy X-ray absorptiometry. J Oral Sci 2016;58:185-94

14. Mostafa RA, Arnout EA, Abo El-Fotouh MM. Feasibility of cone beam computed tomography radiomorphometric analysis and fractal dimension in assessment of postmenopausal osteoporosis in correlation with dual X-ray absorptiometry. Dentomaxillofac Radiol 2016;45:20160212.

15. Dagistan S, Bilge OM. Comparison of antegonial index, mental index, panoramic mandibular index and mandibular cortical index values in the panoramic radiographs of normal males and male patients with osteoporosis. Dentomaxillofac Radiol 2010;39:290-4.

16. Al-Dam A, Blake F, Atac A, Amling M, Blessmann M, Assaf A, et al. Mandibular cortical shape index in non-standardised panoramic radiographs for identifying patients with osteoporosis as defined by the German Osteology Organization. J Craniomaxillofac Surg 2013;41:e165-9.

17. Govindraju P, Chandra P. Radiomorphometric indices of the mandible-An indicator of osteoporosis. J Clin Diagn Res 2014;8:195-8.

18. Savic Pavicin I, Dumancic J, Jukic T, Badel T, Badanjak A. Digital orthopantomograms in osteoporosis detection: Mandibular density and mandibular radiographic indices as skeletal BMD predictors. Dentomaxillofac Radiol 2014;43:20130366.

19. Calciolari E, Donos N, Park JC, Petrie A, Mardas N. Panoramic measures for oral bone mass in detecting osteoporosis: A systematic review and meta-analysis. J Dent Res 2015;94:17S-27S. 
20. Benson BW, Prihoda TJ, Glass BJ. Variations in adult cortical bone mass as measured by a panoramic mandibular index. Oral Surg Oral Med Oral Pathol 1991;71:349-56.

21. Gulsahi A, Yüzügüllü B, Imirzalioglu P, Genç Y. Assessment of panoramic radiomorphometric indices in Turkish patients of different age groups, gender and dental status. Dentomaxillofac Radiol 2008;37:288-92.

22. Hastar E, Yilmaz HH, Orhan H. Evaluation of mental index, mandibular cortical index and panoramic mandibular index on dental panoramic radiographs in the elderly. Eur J Dent 2011;5:60-7.

23. Bajoria AA, Ml A, Kamath G, Babshet M, Patil P, Sukhija P, et al. Evaluation of radiomorphometric indices in panoramic radiograph $-\mathrm{A}$ screening tool. Open Dent J 2015;9:303-10.

24. Kim OS, Shin MH, Song IH, Lim IG, Yoon SJ, Kim OJ, et al. Digital panoramic radiographs are useful for diagnosis of osteoporosis in Korean postmenopausal women. Gerodontology 2016;33:185-92.

25. Leite AF, Figueiredo PT, Guia CM, Melo NS, de Paula AP. Correlations between seven panoramic radiomorphometric indices and bone mineral density in postmenopausal women. Oral Surg Oral Med Oral Pathol Oral Radiol Endod 2010;109:449-56.

26. Barngkgei I, Joury E, Jawad A. An innovative approach in osteoporosis opportunistic screening by the dental practitioner: The use of cervical vertebrae and cone beam computed tomography with its viewer program. Oral Surg Oral Med Oral Pathol Oral Radiol 2015;120:651-9.

27. Koh KJ, Kim KA. Utility of the computed tomography indices on cone beam computed tomography images in the diagnosis of osteoporosis in women. Imaging Sci Dent 2011;41:101-6.

28. Devlin H, Karayianni K, Mitsea A, Jacobs R, Lindh C, van der Stelt P, et al. Diagnosing osteoporosis by using dental panoramic radiographs: The OSTEODENT project. Oral Surg Oral Med Oral Pathol Oral Radiol Endod 2007;104:821-8.

29. Jonasson G, Sundh V, Hakeberg M, Hassani-Nejad A, Lissner L, Ahlqwist $\mathrm{M}$, et al. Mandibular bone changes in 24 years and skeletal fracture prediction. Clin Oral Investig 2013;17:565-72.

30. Jagelaviciene E, Krasauskiene A, Zalinkevicius R, Kubilius R, Vaitkeviciene I. The relationship between the calcaneal bone mineral density and the mental index in post-menopausal females. Dentomaxillofac Radiol 2013;42:20120050.

31. Dutra V, Yang J, Devlin H, Susin C. Radiomorphometric indices and their relation to gender, age, and dental status. Oral Surg Oral Med Oral Pathol Oral Radiol Endod 2005;99:479-84. 